\title{
Persistence of Ion Temperature Gradient Turbulent Transport at Finite Normalized Pressure
}

\section{$\operatorname{AUTHOR}(\mathrm{S}):$}

Ishizawa, A.; Urano, D.; Nakamura, Y.; Maeyama, S.; Watanabe, T.-H.

\section{CITATION:}

Ishizawa, A.... [et al]. Persistence of Ion Temperature Gradient Turbulent Transport at Finite Normalized Pressure. Physical Review Letters 2019, 123: 025003.

\section{ISSUE DATE:}

2019-07-10

URL:

http://hdl.handle.net/2433/243217

\section{RIGHT:}

(c) 2019 American Physical Society; 許諾条件に基づいて掲載しています $\circ$ 


\title{
Persistence of Ion Temperature Gradient Turbulent Transport at Finite Normalized Pressure
}

\author{
A. Ishizawa, D. Urano, and Y. Nakamura \\ Graduate School of Energy Science, Kyoto University, Uji 611-0011, Japan \\ S. Maeyama and T.-H. Watanabe \\ Department of Physics, Nagoya University, Nagoya 464-8602, Japan
}

(Received 23 July 2018; revised manuscript received 17 May 2019; published 10 July 2019)

\begin{abstract}
Plasma $\beta$ dependence of electromagnetic turbulent transport is investigated by means of gyrokinetic simulations with self-consistent change of the equilibrium magnetic field. It is found that energy transport due to ion-temperature-gradient (ITG) driven turbulence does not decrease with increasing $\beta$; that is, the ion energy diffusivity does not decrease, and the electron energy diffusivity increases with $\beta$. This is because magnetic fluctuations are significantly influenced by the background magnetic field structure change with $\beta$ by the Pfirsch-Schluter current. The magnetic field change weakens the suppression effect of magnetic perturbations on the growth of the ITG mode, and it also suppresses nonlinear zonal flow production. The influence of the magnetic field change is significant as the global magnetic shear increases.
\end{abstract}

DOI: 10.1103/PhysRevLett.123.025003

Introduction.-A finite value of normalized pressure $\beta$ makes turbulence electromagnetic in magnetized plasmas, and magnetic fluctuations become important with increasing $\beta$ [1-4]. The understanding of magnetic fluctuations in turbulence is an important issue in magnetic confinement and in space and astrophysics [5,6]; for instance, magnetic fluctuations have a significant impact on the normalized pressure $\beta$ dependence of electromagnetic turbulent transport, which is one of the central issues in magnetized fusion plasma research, because it is linked to the fusion reaction rate and also related to the bootstrap current production that is important for steady state operation of tokamaks. Magnetic fluctuations influence not only the linear growth rate of instabilities but also the nonlinear saturation level of turbulence because nonlinear interactions in terms of the Maxwell stress become active. As a consequence, electromagnetic turbulence is not as well understood as electrostatic turbulence, which corresponds to the low- $\beta$ limit.

Toroidal magnetic confinement systems exhibit nonuniformity of the vacuum magnetic field strength, which is strong at the inside and weak at the outside of the torus, and this nonuniformity causes drift-wave instabilities such as the toroidal ion temperature gradient (ITG) mode, trapped electron mode (TEM), and kinetic ballooning mode $(\mathrm{KBM})$. Nonlinear gyrokinetic simulation studies demonstrate the $\beta$ dependence of turbulent transport due to these instabilities in tokamak and helical or stellarator plasmas [1-4]. The linear growth rate of the ITG mode is suppressed with increasing $\beta$ because of magnetic fluctuations, and turbulent transport is reduced with $\beta$ as well. The suppression of the ITG turbulence leads to a dominance of TEM at finite $\beta$ for cyclone base case (CBC) parameters.
When $\beta$ is further increased, turbulence can be driven by $\mathrm{KBM}$, which is a MHD-like instability modified by driftwave and finite Larmor radius (FLR) effects. The nonlinear turbulent state driven by these electromagnetic instabilities is influenced by magnetic fluctuations. For instance, the zonal flow amplitude, which controls the level of ITG turbulence, is suppressed by stochastic magnetic fields [7]. The magnetic stochasticity is produced by energy transfer from unstable ballooning parity modes to stable tearing parity modes, and then influences the amplitude of the ITG turbulence [8]. Even though these nonlinear effects are included, the turbulent transport decreases with increasing $\beta$ in the previous work [1-4]. On the other hand, experimental studies show no clear trends of $\beta$ dependencies of confinement [9-13], and their tendencies of $\beta$ scaling are contradictory. Thus, further understanding of the $\beta$ dependence of turbulent transport is required, necessitating more modeling [14].

Past research, as cited above, has focused on turbulence driven by instabilities due to toroidicity of the vacuum field. However, in addition to the vacuum field, the field structure is changed by the presence of plasma in toroidal systems through the Pfirsch-Schluter current, which is proportional to $\beta$. The $\beta$ dependence of MHD instabilities is significantly influenced by the magnetic field configuration change due to the Pfirsch-Schluter current, suggesting that the field change can have an impact on the $\beta$ dependence of electromagnetic drift-wave turbulence as well as MHD stability. From this point of view the influence of the magnetic field change on the drift-ballooning mode (DBM) is studied [15]. On the other hand, in the previous gyrokinetic simulations on the ITG turbulence [1-4], $\beta$ 
is the controlling parameter of the amount of magnetic perturbations, because $\beta$ appears only in Ampere's law of the normalized gyrokinetic-Maxwell system. It is noted that some of these simulations have included the pressure gradient term, which depends on $\beta$, in the curvature drift velocity. In toroidal plasmas, however, finite $\beta$ influences the magnetic field configuration through the production of the Pfirsch-Schluter current resulting in the Shafranov shift of the magnetic axis, and the local magnetic shear $\hat{s}$ deviates from the global magnetic shear $s$. A minimal model describing the change of magnetic field is the $s-\alpha$ model [16], which is based on the Shafranov equilibrium and on the large aspect ratio approximation. The model is widely used to investigate not only ballooning modes but also drift-wave instabilities [17-20].

In this Letter, we investigate the $\beta$ dependence of electromagnetic turbulent transport while taking into account the change of magnetic field structure, using the gyrokinetic simulation code GKV $[21,22]$. We will demonstrate that the energy diffusivity due to the ITG turbulence does not decrease with increasing $\beta$, and this influence of magnetic field change is significant as the global magnetic shear increases. The enhancement of turbulent transport is caused by a change of the turbulent magnetic fluctuations due to the field change by $\beta$.

Simulation model. - We take into account finite $\beta$ modifications of the magnetic field configuration by the PfirschSchluter current in terms of the $s-\alpha$ model in flux tube coordinates $(x, y, z)$, where $x=\left[\left(q_{0}\right) /\left(B_{0} r_{0}\right)\right] \psi, \quad y=$ $\left[\left(r_{0}\right) /\left(q_{0}\right)\right]\left(q_{0} \theta-\zeta\right)$, and $z=\theta$. The Pfirsch-Schluter current causes a pressure modulation of the local magnetic shear $\hat{s}=s-\alpha \cos z$, so that $k_{\perp}^{2}=k_{y}^{2}\left\{1+\left[s\left(z-\theta_{k}\right)-\right.\right.$ $\left.\alpha \sin z]^{2}\right\}$ and $\omega_{\mathrm{ds}}=\left(m_{s} v_{\|}^{2}+\mu B\right) \bar{\omega}_{\mathrm{ds}}$ with $\bar{\omega}_{\mathrm{ds}}=\left[\left(-k_{y}\right) /\right.$ $\left.\left(q_{s} R_{0} B\right)\right]\left\{\cos z+\left[s\left(z-\theta_{k}\right)-\alpha \sin z\right] \sin z\right\}$ and $\theta_{k}=-k_{x} /$ $\left(s k_{y}\right)$, where $\alpha=-q_{0}^{2} R_{0} \beta(1 / p)(d p / d r)$ is the pressure modulation parameter. It is remarked that the pressure gradient in the curvature drift velocity is neglected in this work; its effect is discussed in Ref. [23]. Parameters are the same as those for the CBC [1-4], so that $\alpha=35.7 \beta_{i}$, where $\beta_{i}=\beta / 2$ is the normalized ion pressure. In the remainder of this Letter, the magnetic field changed (MFC) and the magnetic field fixed (MFF) cases represent $\alpha \neq 0$ and $\alpha=0$, respectively.

$\beta$ dependence of linear instability.- Figure 1 shows the $\beta$ dependence of the linear growth rate of instabilities for a poloidal wave number $k_{y} \rho_{T i}=0.2$ for MFC and for MFF. The growth rate of the ITG mode decreases with $\beta$, because the stabilizing effect of magnetic perturbations increases. The growth rate for the MFF is the same as that for the $\mathrm{CBC}$ in previous work [1-4]. On the other hand, the growth rate for the MFC is larger than the MFF. Hence, the finite $\beta$ stabilizing effect on the ITG mode is less prominent. A similar $\beta$ dependence of the growth rate of the ITG mode is observed in linear analysis using the Miller local

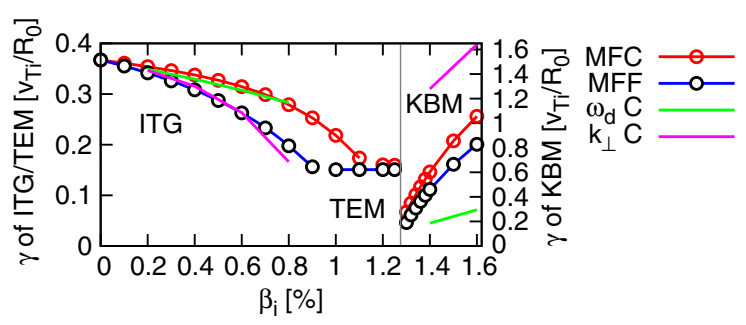

FIG. 1. Linear growth rate as a function of normalized ion pressure $\beta_{i}$ in the magnetic field changed case (MFC, $\alpha \neq 0$ ) and in the magnetic field fixed case (MFF, $\alpha=0)$ at $k_{y} \rho_{T i}=0.2$. The magnetic field changed only in $\omega_{d}\left(\omega_{d} C\right)$ and only in $k_{\perp}^{2}\left(k_{\perp} C\right)$ cases are added.

equilibrium [23,24]. It is remarked that the growth rate of the $\mathrm{KBM}$ is enhanced.

$\beta$ dependence of turbulent transport.- The MFC has significant impact not only on the linear growth rate of the ITG mode but also on the $\beta$ dependence of turbulent transport. Figure 2 shows the $\beta$ dependence of the ion and electron energy diffusivities $\chi_{i}$ and $\chi_{e}$ calculated from nonlinear simulations. The ion energy diffusivity $\chi_{i}$ due to the ITG turbulence decreases with $\beta$ for the MFF, consistent with previous work [1-4]. In contrast, $\chi_{i}$ does not decrease with $\beta$ for the MFC. Furthermore, the electron energy diffusivity $\chi_{e}$ increases with $\beta$ because of the increase of electrostatic and magnetic perturbation amplitudes. Hence, the MFC due to the Pfirsch-Schluter current can make the electromagnetic ITG turbulent transport significant at finite $\beta$. It is remarked that we observed the run-away-nonzonal transition, which is the increase of turbulent fluctuations without saturation at high- $\beta[7,25,26]$, so that we are not able to evaluate $\chi_{i}$ and $\chi_{e}$ for $\beta_{i}>0.65 \%$. Thus, the MFC does not influence the threshold of the runaway-nonzonal transition. It is considered that the runaway-nonzonal transition is caused by the suppression of zonal flows by stochastic magnetic field [26].

Suppression of zonal flows at finite $\beta$.-We have observed that the turbulent diffusivity does not decrease for the MFC, and is larger than the MFF, so we expect that the partition of zonal flow energy is smaller in the MFC. That is confirmed by the spectra of electrostatic potential at $\beta_{i}=0.65 \%$ in Fig. 3(a). The peak of the ITG turbulence is located at $k_{y} \rho_{T i}=0.15$, and the zonal component is at

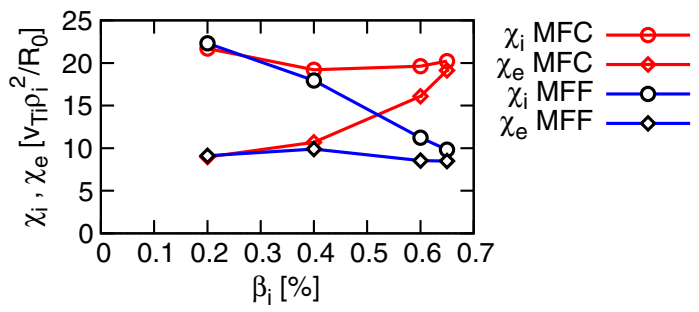

FIG. 2. Ion and electron energy diffusivity in the MFC and MFF as a function of $\beta_{i}$. 

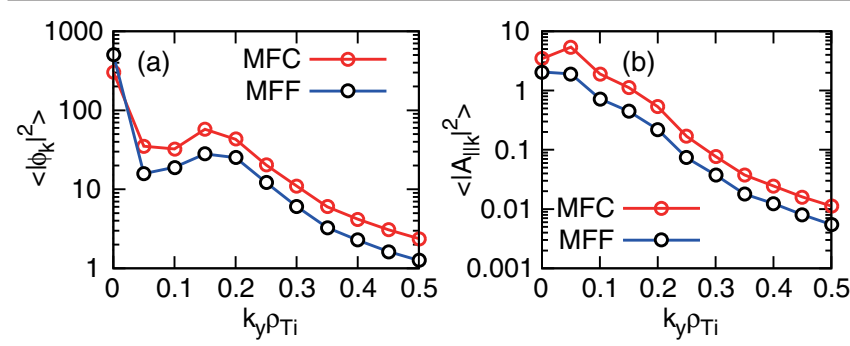

FIG. 3. Poloidal wave number spectra of (a) electrostatic potential $\left\langle\left|\phi_{k}\right|^{2}\right\rangle$ and (b) vector potential $\left\langle\left|A_{\| k}\right|^{2}\right\rangle$ for MFC and MFF at $\beta_{i}=0.65 \%$.

$k_{y} \rho_{T i}=0$. The zonal flows are suppressed and the amplitude of the ITG turbulence is enhanced for the MFC. Thus, the partition of zonal flow energy is smaller in the MFC, and the smaller zonal flow energy partition is linked to the enhancement of the ITG turbulence. This explains the enhancement of turbulent transport for the MFC from the MFF in Fig. 2. The difference between zonal flow partitions is attributed to nonlinear entropy transfer producing zonal flows because the zonal flow residual levels for the MFC and MFF are the same. The zonal component of the entropy balance equation

$$
\frac{d}{d t}\left(\sum_{s} S_{s}+W_{e s}+W_{e m}\right)_{\mathrm{zf}}=T_{\mathrm{zf}}+D_{\mathrm{zf}}
$$

implies that the nonlinear entropy transfer $T_{\mathrm{zf}}$ from turbulence produces a zonal component of the entropy variable $S_{s}$, the polarization electrostatic energy $W_{e s}=$ $\left[\sum_{s}\left(1-\Gamma_{0 s}\right)|\phi|^{2}\right]_{\mathrm{zf}}$, and the magnetic energy $W_{e m}$, and the collisional dissipation is represented by $D_{\mathrm{zf}}$ [4]. We evaluated the time integration of nonlinear transfer to zonal component over the entire simulation $\int T_{\mathrm{zf}} d t$, and found that $T_{\mathrm{zf}}$ for the MFC is 1.8 times as large as in the MFF, and thus the nonlinear production of the zonal component of $\sum_{s} S_{s}+W_{e s}+W_{e m}$ does not explain the larger zonal flows in the MFF. Thus, we investigate the details of the balance equation and extract the polarization electrostatic energy $W_{e s}$ part of Eq. (1) to directly evaluate zonal flow production:

$$
\frac{d}{d t}\left[W_{e s}\right]_{\mathrm{zf}}=N_{\mathrm{zf}}+L_{\mathrm{zf}}+D_{\mathrm{zf}}^{\prime}
$$

derived from the 0th moment of the gyrokinetic equation, where $N_{\mathrm{zf}}=\left[\phi^{*} \sum_{s} \int\left(\phi J_{0 s}, \delta f_{s}\right) J_{0 s} d^{3} v\right]_{\mathrm{zf}}-$ $\left[\phi^{*} \sum_{s} \int\left(v_{\|} A_{\|} J_{0 s}, \delta f_{s}\right) J_{0 s} d^{3} v\right]_{\mathrm{zf}}$. We refer to the first term as the Reynolds stress and to the second term as the Maxwell stress, as they have direct counterparts in the reduced MHD equations in the long-wavelength limit. It is noted that we have a new term $L_{z f}$, which comes from linear terms in the 0th moment equation and represents the transfer from the zonal entropy and zonal magnetic field

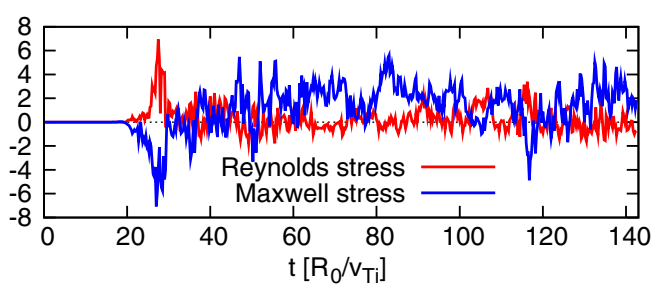

FIG. 4. Time history of zonal flow production by the Reynolds and Maxwell stresses for the MFC at $\beta_{i}=0.65 \%$.

energy $\left[\sum_{s} S_{s}+W_{e m}\right]_{\mathrm{zf}}$. Figure 4 shows that the Reynolds stress drives zonal flows but is counteracted by the Maxwell stress at the initial saturating phase of the turbulence, which occurs from $t \approx 20$ to $t \approx 40$. A part of this initial saturation process may be related to the secondary (Kelvin-Helmholtz) instability and its suppression by the field line bending stabilization [15]. However, this initial saturation process does not control the amplitude of zonal flow in the quasisteady state. The Reynolds stress becomes weak and the Maxwell stress dominantly drives the zonal flows at the later saturation phase in Fig. 4. Table I shows the time averaged contribution of each term to the production of zonal flows at $\beta_{i}=0.65 \%$. Each term is integrated over two time regimes: the initial saturation onset phase, and the entire simulation. In the saturation onset phase, the Reynolds stress, which drives the zonal flows, is larger than the Maxwell stress, which counteracts the Reynolds stress, and thus we observe net zonal flows production. The contribution of the linear terms is small. On the other hand, when we integrate each term over the entire simulation, all of these terms produce zonal flows, and the Maxwell stress dominates the nonlinear production. The Maxwell stress and the transfer from $\left[\sum_{s} S_{s}+W_{e m}\right]_{\mathrm{zf}}$ in the MFF is larger than in the MFC. Hence, the suppression of the zonal flows in the MFC comes from the reduction of the Maxwell stress and the transfer from $\left[\sum_{s} S_{s}+W_{e m}\right]_{\mathrm{zf}}$ in a later saturation phase. The Maxwell stress includes the influence of magnetic stochasticity on zonal flows. The magnetic stochasticity reduces the zonal flow amplitude [27,28], and the suppression effect can be

TABLE I. Zonal flow production by the Reynolds and Maxwell stresses, their sum, and the transfer from other zonal components (FOZC) $\left[\sum_{s} S_{s}+W_{e m}\right]_{\mathrm{zf}}$, integrated over the initial saturating phase and integrated over the entire simulation, for the MFC and MFF at $\beta_{i}=0.65 \%$. Values are normalized to the total stress for

\begin{tabular}{|c|c|c|c|c|}
\hline Zonal flow production & Reynolds st. & Maxwell st. & Total & FOZC \\
\hline Saturating phase (MFC) & 2.23 & -1.23 & 1. & 0.21 \\
\hline Saturating phase (MFF) & 2.51 & -1.71 & 0.80 & 0.31 \\
\hline Entire (MFC) & 0.21 & 0.79 & 1. & 0.81 \\
\hline Entire (MFF) & 0.29 & 0.93 & 1.21 & 1.1 \\
\hline
\end{tabular}
the MFC. 
enhanced by the MFC, because the amplitude of the magnetic perturbations is enhanced as shown in Fig. 3(b).

Discussion of ITG enhancement.-Here, we discuss the mechanism of the enhancement of the linear growth rate of the ITG mode. It is commonly understood that a larger perpendicular wave number $k_{\perp}^{2}$ has a stabilizing effect on microinstabilities through FLR effects, and a larger magnetic drift frequency $\omega_{d}$ with negative sign has a destabilizing effect. Since both of them are suppressed by the MFC compared to the MFF, it is expected that the impact of $\alpha$ on $k_{\perp}^{2}\left(\omega_{d}\right)$ enhances (reduces) the growth rate of microinstabilities. That is confirmed by the enhancement of the growth rate of the KBM for the changed magnetic field only in $k_{\perp}^{2}$ case $\left(k_{\perp} C\right)$ and by the suppression of the KBM for the changed field only in $\omega_{d}$ case $\left(\omega_{d} C\right)$ in Fig. 1 . The destabilizing effect overcomes the stabilizing effect, and thus the sum of them enhances the growth rate of the KBM. This enhancement of the KBM corresponds to the enhancement of the DBM in Ref. [15]. However, this consideration is not helpful for understanding the enhancement of the growth rate of the ITG mode in the MFC, because the growth rate is similar to the MFF (MFC) when the magnetic field is changed only in $k_{\perp}^{2}\left(\omega_{d}\right)$ as shown by the lines labeled with $k_{\perp} C\left(\omega_{d} C\right)$ in Fig. 1. Next, we compare the electromagnetic calculation with the corresponding electrostatic one. The ratio of the growth rate in the MFC to that in the MFF is small in the electrostatic calculation $\gamma_{\mathrm{MFC}} / \gamma_{\mathrm{MFF}}=1.08$ compared to the electromagnetic one $\gamma_{\mathrm{MFC}} / \gamma_{\mathrm{MFF}}=1.41$ for $\beta_{i}=0.8 \%$ and $k_{y} \rho_{T i}=0.2$. This implies that magnetic perturbations are responsible for the enhancement of growth rate for the MFC. Since the magnetic perturbation is linked to the electron parallel velocity through Ampere's law, we examine the linearized equation of the electron parallel velocity

$$
\begin{aligned}
\frac{\partial u_{\| e}}{\partial t}= & -v_{T e} \nabla_{\|} p_{\| e}+\frac{v_{T e}}{T_{e}}\left(\nabla_{\|} \phi+\frac{\partial A_{\|}}{\partial t}\right) \\
& -i \bar{\omega}_{\mathrm{de}}\left(q_{\| e}+q_{\perp e}+4 u_{\| e}\right)+v_{T e}\left(p_{\| e}-p_{\perp e}\right) \nabla_{\|} \ln B \\
& +i \bar{\omega}_{* \mathrm{e}}\left(1+\eta_{e}\right) \frac{v_{T e}}{T_{e}} A_{\|},
\end{aligned}
$$

where $\bar{\omega}_{* \mathrm{e}}=\left[\left(k_{y}\right) /\left(L_{n} B\right)\right]$, and electron FLR effects are neglected. The equation suggests that the kinetic electron effects and magnetic perturbations violate the adiabatic response approximation. The absolute values of the balance between the $\nabla_{\|} \phi$ and $\nabla_{\|} p_{e}$ terms, of the $\omega_{* e}$ term, and of the $\omega_{d e}$ term for the linear eigenfunction are plotted as a function of the coordinate along the field line $z$ in Fig. 5. The influence of the $\omega_{* e}$ term is smaller in the MFC than in the MFF around the peak, and the $\omega_{* e}$ term is considered to have a stabilizing effect on the ITG mode [29], so that the stabilizing effect of the $\omega_{* e}$ term is suppressed in the MFC. This is a possible explanation of the higher linear growth rate of the ITG mode in the MFC.

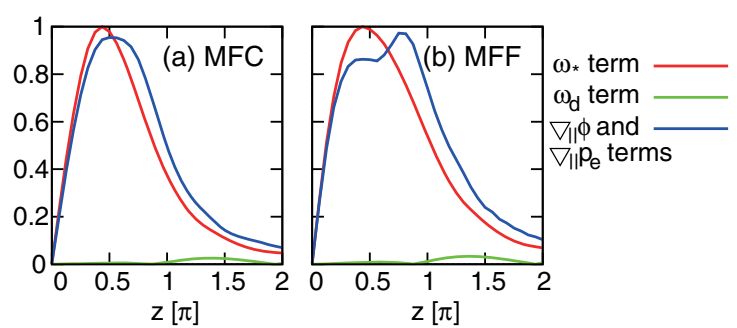

FIG. 5. Absolute values of the $\omega_{* e}$ term, the $\omega_{d e}$ term, and the balance between the $\nabla_{\|} \phi$ and $\nabla_{\|} p_{e}$ terms in the electron parallel velocity equation for the linear eigenfunction at $k_{y} \rho_{T i}=0.2$ and $\beta_{i}=0.8 \%$ for (a) the MFC and (b) the MFF.

Finally, we discuss simulation results for other plasmas obtained by using the $s-\alpha$ model. We found that the MFC effect is significant when the global magnetic shear is finite $s \approx 1$, whereas the effect is weak when $s \ll 1$. Figure 6(a) shows the ratio between the linear growth rate of instability for MFF and that for MFC $\gamma_{\mathrm{MFF}} / \gamma_{\mathrm{MFC}}$ in JET66404 $(\rho=0.33) \quad[30], \quad J E T 75225 \quad[30]$, and AUG29224 $(\rho=0.5)$ [31] as a function of $\beta_{i}$. All of them are unstable against the ITG mode except for JET75225 at $\rho=0.64$ (JET75225r064) [30], which is unstable against the TEM. The influence of MFC is significant for JET66404 with $s=0.7$ [30], JET75225r064 with $s=1.44$ [30], and AUG29224 with $s=1.08$ [31], whereas the effect is negligible for JET75225 at $\rho=0.33$ with $s=0.16$ (JET75225r033)[30]. The ion energy diffusivity $\chi_{i}$ does not decrease with increasing $\beta_{i}$ for the MFC of AUG29224, whereas it decreases for the MFF as shown in Fig. 6(b). Hence, the MFC effect is significant when the global magnetic shear is finite $s \approx 1$ such as JET66404 with $s=0.7$, AUG29224 with $s=1.08$, and JET75225r064 with $s=1.44$. On the other hand, the MFC effect is weak, when the global magnetic shear is small $s \ll 1$ such as the core plasma of JET hybrid discharge [30,32,33], for instance, JET75225r033 with $s=0.16$. This implies that low global magnetic shear is an important parameter for the electromagnetic stabilization [34-36]. A possible explanation for the efficiency of MFC is the elongation of mode structure along the field line by weak global magnetic shear. This is because the Pfirsch-Schluter current
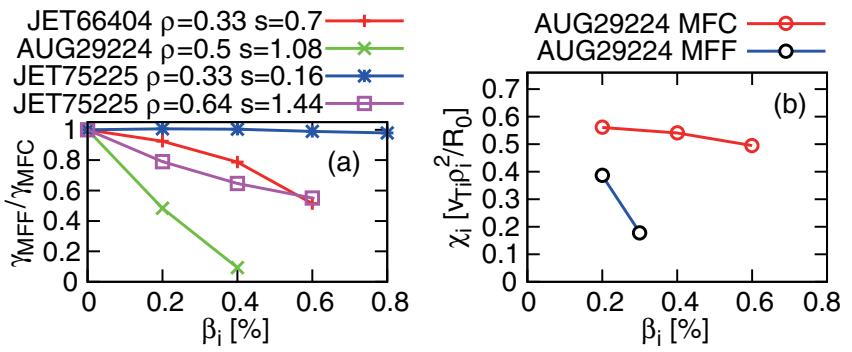

FIG. 6. (a) Ratio between linear growth rate for MFF and that for MFC $\gamma_{\text {MFF }} / \gamma_{\text {MFC }}$ as a function of $\beta_{i}$. (b) Ion energy diffusivity $\chi_{i}$ for MFC and MFF of AUG29224 with $s=1.06$. 
$J_{\mathrm{PS}}=(2 q / B)(d p / d r) \cos \theta$ is proportional to $\cos \theta$, and thus the destabilizing and stabilizing effects on instabilities are averaged out when the mode structure is elongated. It is noted that the MFC effect is significant even when the instability is weak such as the AUG29224 having $\gamma \approx$ $0.03\left[v_{T i} / R_{0}\right]$ (Fig. 6). It is also noted that the elongated mode structure produces nonlinear self-interactions, which may contribute the supplemental reduction of turbulence [4].

Summary.-Investigating the impact of $\beta$ on ITG turbulence, we have shown that the ion energy diffusivity does not decrease and the electron energy diffusivity increases with increasing $\beta$, when the equilibrium magnetic field configuration is changed by the Pfirsch-Schluter current. This is a consequence of the magnetic field change enhancing the linear growth rate of the ITG mode and suppressing the zonal flow activity. The present results imply a bigger importance of ITG driven turbulent transport at finite $\beta$. We also found that the influence of MFC is significant as the global magnetic shear increases, in other words, the electromagnetic stabilization is prominent for low global magnetic shear. This suggests an advantage of plasmas which have a wide-region of low global magnetic shear at the core such as the JET hybrid discharges and their better $\beta$ dependence of confinement [13]. The level of ITG turbulence does not decrease with $\beta$ because the magnetic field change suppresses the stabilizing effect of magnetic perturbations on the ITG mode, resulting in an enhancement of the linear growth rate. The MFC also reduces the zonal flow level by reducing the Maxwell stress and the transfer from the zonal components of the entropy and magnetic energy, which dominantly produce zonal flows in the quasisteady turbulent state at finite $\beta$. It is remarked that we have obtained similar results by taking into account the MFC due to finite $\beta$ to greater accuracy by using the VMEC code. We will report details of these results and also the dependence on profiles, which can influence the $\beta$ dependence of turbulent transport [37], in a separate paper.

A. I. would like to thank Dr. M. J. Pueschel and Professor Y. Kishimoto for their valuable comments. This work is carried out with the support and under the auspices of the NIFS Collaboration Research program (NIFS18KNWT001).

[1] J. Candy, Phys. Plasmas 12, 072307 (2005).

[2] M. J. Pueschel, M. Kammerer, and F. Jenko, Phys. Plasmas 15, 102310 (2008).

[3] M. J. Pueschel and F. Jenko, Phys. Plasmas 17, 062307 (2010).

[4] A. Ishizawa, S. Maeyama, T.-H. Watanabe, H. Sugama, and N. Nakajima, J. Plasma Phys. 81, 435810203 (2015).

[5] G. G. Howes, J. M. TenBarge, W. Dorland, E. Quataert, A. A. Schekochihin, R. Numata, and T. Tatsuno, Phys. Rev. Lett. 107, 035004 (2011).
[6] M. J. Pueschel, F. Jenko, D. Told, and J. Buchner, Phys. Plasmas 18, 112102 (2011).

[7] M. J. Pueschel, P. W. Terry, F. Jenko, D. R. Hatch, W. M. Nevins, T. Gorler, and D. Told, Phys. Rev. Lett. 110, 155005 (2013).

[8] D. R. Hatch, M. J. Pueschel, F. Jenko, W. M. Nevins, P. W. Terry, and H. Doerk, Phys. Rev. Lett. 108, 235002 (2012).

[9] H. Urano, T. Takizuka, H. Takenaga, N. Oyama, Y. Miura, and Y. Kamada, Nucl. Fusion 46, 781 (2006).

[10] L. Vermare, F. Ryter, C. Angioni, A. G. Peeters, J. Stober, R. Bilato, L. D. Horton, B. Kurzan, C. F. Maggi, H. Meister, J. Schirmer, G. Tardini (The ASDEX Upgrade Team), Nucl. Fusion 47, 490 (2007).

[11] C. C. Petty, Phys. Plasmas 15, 080501 (2008).

[12] D. C. McDonald, L. Laborde, J. C. Deboo, F. Ryter, M. Brix, C. D. Challis, P. de Vries, C. Giroud, D. Howell, E. Joffrin, T. C. Luce, J. Mailloux, V. Pericoli-Ridolfini, A. C. C. Sips, K. Thomsen (JET EFDA Contributors), Plasma Phys. Controlled Fusion 50, 124013 (2008).

[13] C. D. Challis et al., Nucl. Fusion 55, 053031 (2015).

[14] G. G. Whelan, M. J. Pueschel, and P. W. Terry, Phys. Rev. Lett. 120, 175002 (2018).

[15] B. N. Rogers and J. F. Drake, Phys. Rev. Lett. 79, 229 (1997).

[16] J. W. Connor, R. J. Hastie, and J. B. Taylor, Phys. Rev. Lett. 40, 396 (1978).

[17] J. Y. Kim, W. Horton, and J. Q. Dong, Phys. Fluids B 5, 4030 (1993).

[18] A. Hirose, L. Zhang, and M. Elia, Phys. Rev. Lett. 72, 3993 (1994).

[19] X. Lapillonne, S. Brunner, T. Dannert, S. Jolliet, A. Marinoni, L. Villard, T. Gorler, F. Jenko, and F. Merz, Phys. Plasmas 16, 032308 (2009).

[20] G. M. Staebler, Nucl. Fusion 58, 115001 (2018).

[21] T.-H. Watanabe and H. Sugama, Nucl. Fusion 46, 24 (2006).

[22] A. Ishizawa, S. Maeyama, T.-H.Watanabe, H. Sugama, and N. Nakajima, Nucl. Fusion 53, 053007 (2013).

[23] N. Joiner, A. Hirose, and W. Dorland, Phys. Plasmas 17, 072104 (2010).

[24] R. L. Miller, M. S. Chu, J. M. Greene, Y. R. Lin-Liu, and R. E. Waltz, Phys. Plasmas 5, 973 (1998).

[25] R. E. Waltz, Phys. Plasmas 17, 072501 (2010).

[26] M. J. Pueschel, P. W. Terry, and D. R. Hatch, Phys. Plasmas 21, 055901 (2014).

[27] P. W. Terry, M. J. Pueschel, D. Carmody, and W. M. Nevins, Phys. Plasmas 20, 112502 (2013).

[28] Z. R. Williams, M. J. Pueschel, P. W. Terry, and T. Hauff, Phys. Plasmas 24, 122309 (2017).

[29] A. Hirose, Phys. Plasmas 7, 433 (2000).

[30] J. Citrin et al., Plasma Phys. Controlled Fusion 57, 014032 (2015).

[31] H. Doerk, M. Dunne, F. Jenko, F. Ryter, P. A. Schneider, and E. Wolfrum, Phys. Plasmas 22, 042503 (2015).

[32] J. Garcia, C. Challis, J. Citrin, H. Doerk, G. Giruzzi, T. Görler, F. Jenko, and P. Maget, Nucl. Fusion 55, 053007 (2015).

[33] H. Doerk, C. Challis, J. Citrin, J. Garcia, T. Görler, and F. Jenko, Plasma Phys. Controlled Fusion 58, 115005 (2016). 
[34] J. Citrin, F. Jenko, P. Mantica, D. Told, C. Bourdelle, J. Garcia, J. W. Haverkort, G. M. D. Hogeweij, T. Johnson, and M. J. Pueschel, Phys. Rev. Lett. 111, 155001 (2013).
[35] P. Mantica et al., Phys. Rev. Lett. 102, 175002 (2009).

[36] P. Mantica et al., Phys. Rev. Lett. 107, 135004 (2011).

[37] G. G. Whelan and M. J. Pueschel (private communication). 